\title{
„Die unerträgliche Geschichte des Vergleiches zwischen geistiger Behinderung und Animalität (und der Versuch sie hinter sich zu lassen)“
}

\author{
Alice Crary
}

(C) Der/die Autor(en) 2018

Wie sollen wir mit der langen und beschämenden Geschichte von Tiervergleichen umgehen, die ein wesentliches Element der Rhetorik der sozialen Marginalisierung, des Missbrauchs und der Tötung von geistig behinderten Menschen darstellt? Gibt es angemessene Antworten auf diese Geschichte, Antworten, die uns die Mittel an die Hand geben, um auf der moralischen Gleichheit von geistig behinderten Menschen zu insistieren, ohne dabei die Herabwürdigung von Tieren in unserem politischen und moralischen Diskurs einfach zu wiederholen? Ist es möglich, ein Bild von Tieren als intrinsisch moralisch wertvoll mit dem Einstehen für die Gleichheit der menschlichen Würde zu verbinden? Bei der Beantwortung dieser Fragen ist es selbstverständlich, die Schriften von Peter Singer, einem international anerkannten und zugleich äußerst kontrovers diskutierten Philosophen, heranzuziehen. Während Singer auf der einen Seite als Protagonist der zeitgenössischen Tierethik gilt und in diesem Kontext mit seinen Publikationen die relevanten Diskussionen um den moralischen Status von Tieren nicht nur inhaltlich angestoßen, sondern auch maßgeblich in ihren zentralen Aspekten bestimmt hat, hat er auf der anderen Seite zugleich heftigen Widerspruch - ja sogar Widerstand - für seine Thesen erhalten. Empfindlich getroffen fühlen sich viele Menschen vor allem auch von seinen Vergleichen zwischen bestimmten Tierarten und Menschen mit geistigen Behinderungen.

This article by Alice Crary was the topic of a session of the Ira W. DeCamp Bioethics Seminar at Princeton University's Center for Human Values, on Tuesday, November 28, 2017—a session at which the philosophers Peter Singer and Eva Feder Kittay were the respondents. The article has been published in Lori Gruen and Fiona Probyn Rapsey, eds., Animaladies, Bloomsbury, 2018. Below is a paraphrase of the article by Dagmar Borchers and Alice Crary followed by revised versions of Singer's and Kittay's original responses to it, as well as by Crary's subsequent response to Singer and Kittay.

A. Crary $(\square)$

Regent's Park College, University of Oxford, Oxford, Großbritannien

E-Mail: alice.crary@regents.ox.ac.uk 
Hintergrund dieser Vergleiche bei Singer sind im Wesentlichen seine Argumente gegen das Laster, das er „Speziesismus“ nennt. Wenn es um die Frage nach dem moralischen Status von Tieren geht, hält Singer all jene Positionen für argumentativ unhaltbar, die Menschen allein aufgrund ihrer Zugehörigkeit zur Spezies Mensch moralische Relevanz zusprechen möchten. Als Utilitarist und Pathozentriker plädiert er stattdessen dafür, als entscheidendes Kriterium der moralischen Relevanz die Leidensfähigkeit, also die Fähigkeit, Schmerz und Schaden zu erfahren, auszuweisen. Nur Wesen, die diese Fähigkeit haben, können das Interesse ausbilden, nicht zu leiden. Insofern es beim moralischen Handeln darum geht, von einem unparteiischen Standpunkt aus zu urteilen, soll Singer zufolge grundsätzlich zunächst einmal nur zählen, wie gro $\beta$ das jeweilige Ausmaß des Schadens bzw. Leidens wäre. Es gilt das Prinzip der gleichen Interessenabwägung.

Singer hat bei vielen Gelegenheiten immer wieder Vergleiche zwischen geistig behinderten Menschen und Tieren gezogen. Auch wenn er mehrfach betont hat, dass es ihm dabei nicht darum geht, geistig Behinderte herabzusetzen, sondern die moralische Position von Tieren zu stärken, sind diese Vergleiche der Sache nach fragwürdig und zudem für viele Menschen äußerst verletzend. Problematisch ist vor allem auch die Tradition, auf die man in Bezug auf diese Vergleiche verweisen kann: Es lässt sich u.a. zeigen, dass bereits im 19. Jahrhundert in England Verbindungen dieser Art formuliert wurden. So hat zum Beispiel Charles Darwin in seiner Schrift „The Descent of Man“ von 1871, auf der Suche nach der ihm fehlenden Verbindung zwischen Menschen und seinen Vorfahren, den Primaten, über Verwandtschaftsbeziehungen zwischen den am höchsten entwickelten Affen und-wie er es sich vorgestellt hat—den am ,niedrigsten“ entwickelten Menschen spekuliert. Hintergrund war sein Bestreben, einen monogenetischen Stammbaum des Menschen nachzuzeichnen. Die Evolutionstheorie hat dann im Verlauf des 19. und frühen 20. Jahrhunderts zusammen mit der sich rasant entwickelnden Genetik den Boden für jene Eugeniker und Rassisten bereitet, die als Vorläufer nationalsozialistischen Gedankenguts gelten können. So finden sich u.a. bei Ernst Haeckel Ideen zur Steuerung der natürlichen Selektion durch Sterilisation und Isolation von behinderten Menschen sowie auch zu deren Tötung in speziellen Fällen. Auch in den USA lassen sich bis zum 2. Weltkrieg politische Tendenzen in diese Richtung ausmachen, mit Maßnahmen wie Einwanderungsbeschränkungen, Heiratsverboten, Zwangssterilisationen und Deportationen. Die ideologischen Fundamente, auf denen die Nationalsozialisten ihre Propaganda und ihre menschenverachtenden politischen Maßnahmen gründen konnten, waren also zumindest in Teilen in Europa und den USA bereits gelegt.

Singer ist selbstredend nicht in diesem Kontext zu sehen. Ihm geht es darum, eine vorurteilsfreie, auf Experiment und Beobachtung beruhende, mithin also empirisch gestützte Differenzierung menschlicher und tierlicher Fähigkeiten vorzulegen, die prinzipiell moralisch relevant sind. Möglichst frei von Vorurteilen möchte er mittels empirischer Belege Anhaltspunkte für moralisch relevante Aspekte des Mensch- und Tierseins erfassen. Das pure Faktum des Mensch-Seins selbst ist dabei moralisch indifferent. Aus seiner Perspektive sind die Vergleiche zwischen Tieren und Behinderten insofern statthaft, als es darum geht, nach theoretisch ausgewiesenen Gründen 
für moralische Relevanz Ausschau zu halten, die ihrerseits empirisch fundiert und akkurat beschrieben sind.

Aber stimmt Singers eigene Einschätzung? Seine Vergleiche werden von Menschen, auch von Philosophinnen und Philosophen als problematisch und verletzend empfunden. So hat es 2008 bei einer Konferenz am Institut für Philosophie der Stony Brook University in New York eine Auseinandersetzung zwischen Peter Singer und Eva Feder Kittay gegeben. Kittay ist eine prominente Philosophin und Feministin und zudem Mutter einer geistig behinderten Tochter namens Sesha. Für Kittay war der Vergleich nicht nur philosophisch unhaltbar, sondern auch persönlich verletzend. Philosophisch wurde in dieser Kontroverse vor allem Singers Neutralitätsthese in Zweifel gezogen: Was soll es heißen, neutral und unvoreingenommen die empirisch aufweisbaren, moralisch relevanten Eigenschaften von Menschen und Tieren zu eruieren? Kann man neutral etwas adäquat wahrnehmen und in seiner spezifischen Seinsweise überhaupt erfassen? Entgehen einem nicht gerade die relevanten Aspekte des So-Seins von Wesen, wenn man sich nicht auf sie einlässt, sondern wissenschaftlich distanziert auf sie blickt?

Kittay vetrat in dem Disput mit Singer die These, dass bestimmte Arten affektiver Reaktion notwenig und angemessen seien, um zum Beispiel die relevanten Aspekte des Lebens ihrer Tochter zu begreifen. Sie lud Singer ein, ihre Tochter zu besuchen, forderte ihn aber auch auf, Zeit zu investieren, sich einzulassen und eine zugewandte Haltung zu zeigen. Singer stellte ihr im Gegenzug die Frage, welche moralisch relevante Eigenschaft es denn genau sein würde, die in diesem Fall für ihn erkennbar sein und einen entscheidenden Einfluss auf das von ihm formulierte Argument haben würde. Seine Reaktion war durchaus vernünftig im Kontext seiner eigenen philosophischen Position, und Kittays Antwort, das hänge davon ab, wie intensiv er sich einlassen würde, muss entsprechend als inadäquat erscheinen.

$\mathrm{Zu}$ fragen ist aber: Was ist, wenn wir die Neutralitäts-Konzeption Singers aufgeben, der zufolge man mit einer quasi naturwissenschaftlichen Neutralität und Distanz die empirisch nachweisbaren Eigenschaften und Fähigkeiten von Mensch und Tier in Erfahrung zu bringen sucht? Kittays These ist, dass man eben nicht ethisch neutral agieren könne, sondern dass man stattdessen eine bestimmte ethische Voreinstellung bzw. Haltung brauche, mit der man in die Situation der Auseinandersetzung mit (geistig behinderten) Menschen und Tieren hineingehen müsse. Hier kann man zur theoretischen Fundierung u. a. auf Ian Hacking verweisen, der - in enger Anlehnung an Wittgensteins Spätphilosophie - ebenfalls betont, dass die relevanten Konzepte zur Beschreibung des menschlichen Verstandes ethische seien. Ohne eine Konzeption des Guten Lebens für das Gegenüber bereits vorauszusetzen, können wir es mit seinen besonderen geistigen Fähigkeiten nicht adäquat erfassen und verstehen. Wir müssen bereit sein, uns auf die spezifischen Vorstellungen vom Guten Leben einzulassen. Insofern kann man Kittays Position recht geben: Wir müssen auf Individuen durch die Linse einer ethischen Konzeption des Guten Lebens schauen. Um Seshas Leben richtig zu verstehen, müssen wir sie im Lichte einer ethisch angereicherten Perspektive auf die menschliche Existenz betrachten. Wir müssen unser Bild, unsere Wahrnehmung immer wieder auf verschiedenen Wegen anreichern und verfeinern, ggf. auch mittels Literatur, journalistischen und künstlerischen Beiträgen, Filmen etc. Es geht dabei grundsätzlich darum, die Menschen aus sich heraus 
zu begreifen - jeder und jede auf ihre Weise, und dabei allen gleichermaßen eine Daseinsberechtigung zuzusprechen, die unbedingt ist. Dieses Prinzip einer generellen Annahme und Zugewandtheit, die nach den spezifischen Umständen einer für dieses Wesen angemessenen Lebensweise fragt, ließe sich dann auch sehr gut auf Tiere übertragen. Es bedeutet im Kern eine Ethik, die von Hierarchisierungen absieht und stattdessen versucht, den Wesen, die mit uns leben, in ihrem Dasein gerecht zu werden. Dies könnte eine Antwort auf die Frage sein, wie wir die schrecklichen Vergleiche zwischen Menschen und Tieren aufgeben und zugleich allen Menschen und auch Tieren jene moralische Relevanz und Rücksichtnahme zukommen lassen können, die ihnen gebührt.

Open Access Dieser Artikel wird unter der Creative Commons Namensnennung 4.0 International Lizenz (http://creativecommons.org/licenses/by/4.0/deed.de) veröffentlicht, welche die Nutzung, Vervielfältigung, Bearbeitung, Verbreitung und Wiedergabe in jeglichem Medium und Format erlaubt, sofern Sie den/die ursprünglichen Autor(en) und die Quelle ordnungsgemäß nennen, einen Link zur Creative Commons Lizenz beifügen und angeben, ob Änderungen vorgenommen wurden. 